\title{
Optimization of the silica glass capillary and rods drawing process
}

\author{
Grzegorz Wójcik \\ Faculty of Chemistry, Maria Curie Sklodowska University in Lublin pl. Marii Curie-Sklodowskiej 2, 20-031 Lublin
}

Received March 21, 2019; accepted March 31, 2019; published March 31, 2019

\begin{abstract}
Diameter fluctuations of silica glass rods and capillaries, during the drawing process have been studied. We investigated the influence of drawing conditions on the quality of capillaries and rods. We fabricated two preforms made from different quality materials. Fabricated preforms were used to draw microstructured fibers.
\end{abstract}

Photonic fiber has many features not offered by conventional optical fibers. Their technology allows to fabricate structures with complex cross-sections, which makes them useful in various applications in telecommunications [1-2] and sensors [3]. Among their special properties we can point out high birefringence [4], photonic band gap propagation [5], high nonlinearity [6], different shapes, size and symmetry [7].

The microstructured preform is usually made with a well- known stack method using basic elements - rods and capillaries [8]. The distribution of these elements in the preform, mostly corresponds to the cross-section geometry in the final fiber, significantly affecting its optical properties. It is a very comfortable situation because in a relatively simple way various structures can be fabricated. At this stage it is extremely important to ensure the highest optical and geometrical quality of basic elements, because no defects or irregularities can be adjusted in the next technological stages.

In our research we decided to investigate the influence of drawing conditions on the quality of rods and capillaries and then its influence on fibers quality. In the first stage, we used commercial pure silica rods F300, the diameter of each rod being $\varphi=15 \mathrm{~mm}$. Small diameter rods were drawn on the drawing tower with a standard configuration. We drew three different sets of small diameter rods, and the diameter of fabricated rods was equal to $\varphi=1000 \mu \mathrm{m}$ while the force of drawing was equal to $\mathrm{F}=20 \mathrm{~N}$. During the process, diameter fluctuations were measured on-line by a contactless optical measuring gauge Anritsu M551A with ensured accuracy $\pm 0.1 \mu \mathrm{m}$. The optical unit was plugged into a paperless recorder BrainChild VR06. We drew the first section of rods with the drawing speed $\mathrm{V}=1.2 \mathrm{~mm} / \mathrm{min}$. At the beginning of the process (see Fig. 1.) short term fluctuations were observed at the level $\pm 5.5 \mu \mathrm{m}$, which corresponds to $0.55 \%$ of the whole diameter. At this same time, long term fluctuations were less than $0.75 \%$ of the rods diameter.
Next, we observed that long term fluctuations increased up to $0.8 \%$ of the rod diameter.

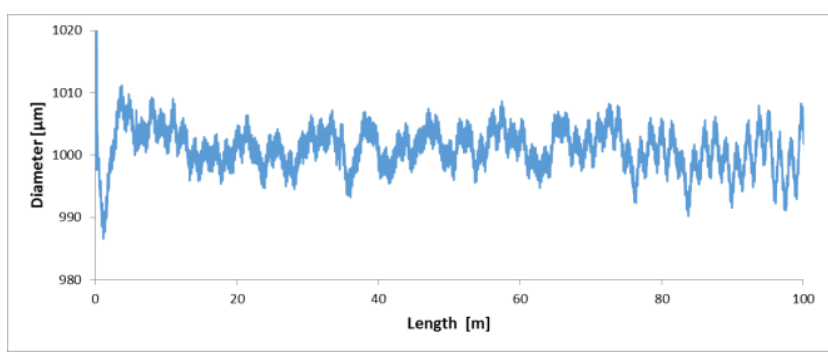

Fig. 1. Rods diameter fluctuations in drawing with the speed $\mathrm{V}=1.2 \mathrm{~m} / \mathrm{min}$.

Next, we set a drawing process with the speed $\mathrm{V}=5 \mathrm{~m} / \mathrm{min}$. In this case, short term fluctuations of the diameter were not higher than $\pm 4 \mu \mathrm{m}(0.4 \%$ of the rod diameter). At the beginning (see Fig. 2) long term fluctuations were very low, next they increased to the value $0.5 \%$. A large increase of the diameter around 32 and 52 meter resulted from the correction of the rod position in the drawing tower oven.

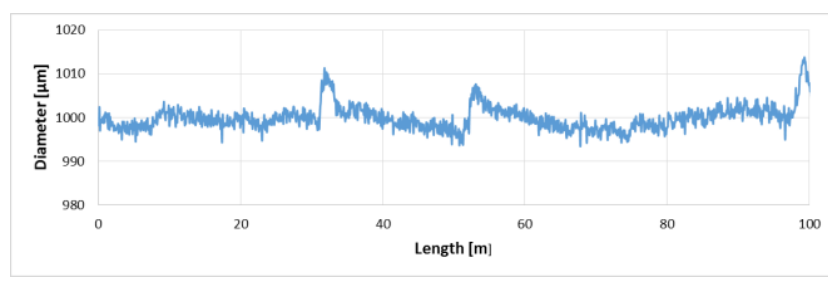

Fig.2. Rods diameter fluctuations in drawing with speed $V=5 \mathrm{~m} / \mathrm{min}$.

The last drawing process was made with the velocity $\mathrm{V}=10 \mathrm{~m} / \mathrm{min}$. During this process we were not able to control the drawing parameters at a correct level. In effect, we had to slow down the process, setting the drawing velocity at $\mathrm{V}=8.2 \mathrm{~m} / \mathrm{min}$. In this case, we registered short term fluctuations around a value of $1 \%$ of the diameter. Long term fluctuations were higher than $2 \%$. 


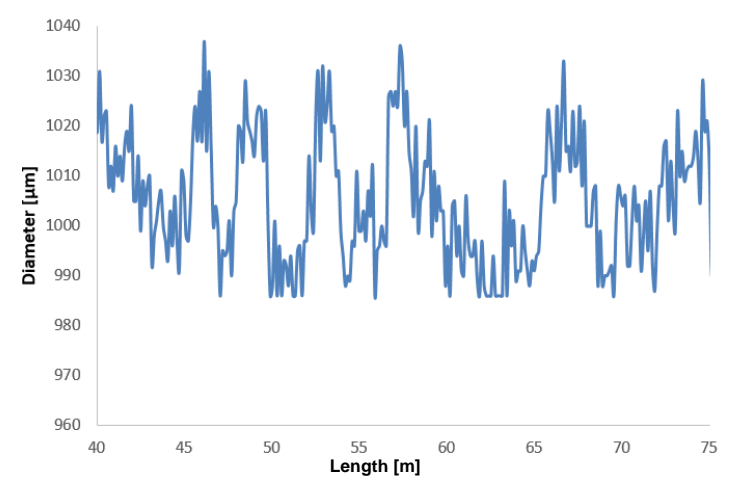

Fig.3. Rod diameter fluctuations in drawing with speed $V=8.2 \mathrm{~m} / \mathrm{min}$.

In the second stage of the investigation, we conducted capillaries drawing from commercial silica tubes HX-S, the outer diameter of tubes equalling $\varphi=27 \mathrm{~mm}$. We drew three sections of capillaries with different drawing speeds. The force of drawing was set at level $\mathrm{F}=30 \mathrm{~N}$, this value allowed to obtain glass soft enough to draw in a stable process and at the same time to compensate an unwanted collapse effect. The inner diameter of capillaries was measured after the drawing process. We drew three sets of capillaries, all sections of which were not shorter than $1=50 \mathrm{~m}$. At the beginning we drew capillaries with the drawing speed equal $\mathrm{V}=1 \mathrm{~m} / \mathrm{min}$. In this case, short term fluctuations (see Fig. 4) were very high. We observed fluctuations higher than $\pm 30 \mu \mathrm{m}$ ( $3 \%$ of total diameter). Around the point $1=40 \mathrm{~m}$ we registered uncontrollable fluctuations of the diameter, which was caused by shifting the tube position in the oven and its correction.

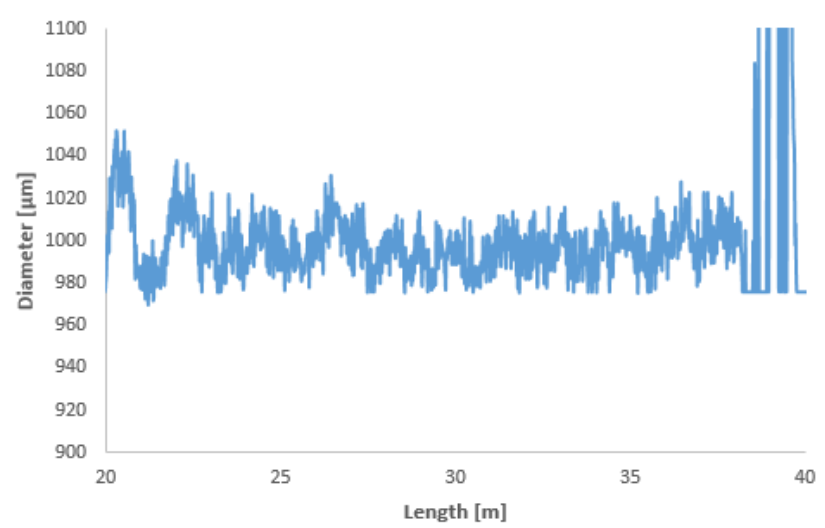

Fig.4. Capillaries diameter fluctuations during drawing with velocity $\mathrm{V}=1 \mathrm{~m} / \mathrm{min}$.

Than we increased the drawing speed up to $\mathrm{V}=5 \mathrm{~m} / \mathrm{min}$. and fabricated next section of capillaries. In this process short term fluctuations were decreased and achieved value $\pm 15 \mu \mathrm{m}$ ( $1.5 \%$ of diameter). Long term fluctuations were measurable and obtained values around $\pm 30 \mu \mathrm{m}$, what corresponded to $3 \%$ of whole diameter (see Fig. 5.).

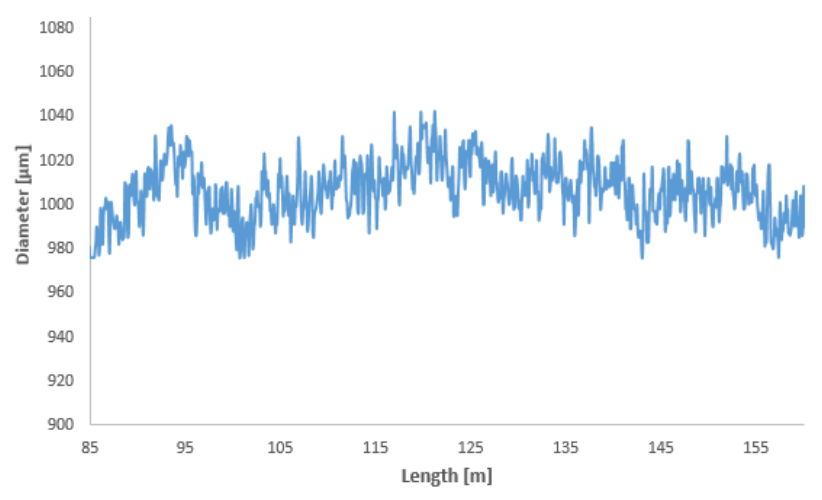

Fig.5. Capillaries diameter fluctuations in drawing with velocity $\mathrm{V}=5 \mathrm{~m} / \mathrm{min}$.

In the last approach, we increased the drawing speed to $\mathrm{V}=10 \mathrm{~m} / \mathrm{min}$. In these conditions we drew the last section of capillaries. In this case we registered the characteristics of diameter fluctuations (see Fig. 6) with the lowest disorders. Short term fluctuations were measured at level $\pm 10 \mu \mathrm{m}$, which corresponded to $1 \%$ of the diameter. Long term fluctuations were also observed and their values were around $15 \mu \mathrm{m}$ ( $1.5 \%$ of diameter).

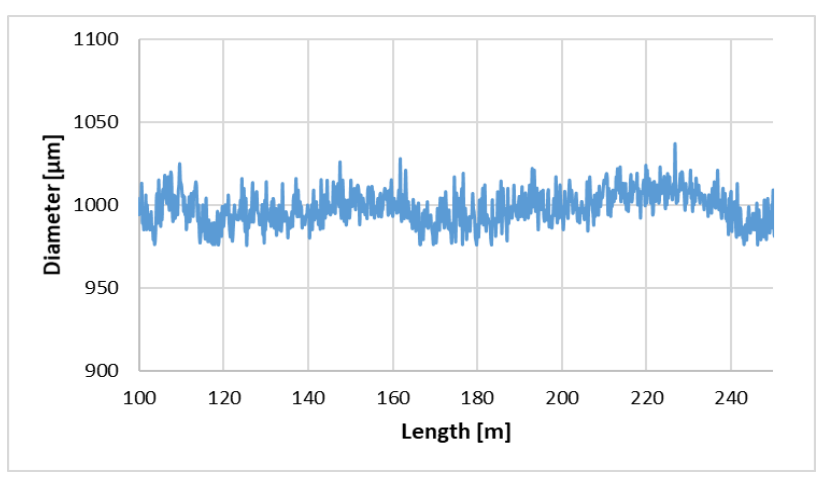

Fig.6. Capilaries diameter fluctuations in drawing with speed $\mathrm{V}=10 \mathrm{~m} / \mathrm{min}$.

Analyses of results obtained during the drawing experiments allowed to select two groups of materials with the smallest and highest diameter fluctuations. These materials were used to fabricate two microstructured preforms. We manufactured preforms with the same type of cross-section - undoped core in the central part, two rings of holes and four rings of rods. In the next step, preforms were stretched to a cane form. In the final fiber we planned to achieve the following geometrical parameters: outer diameter $\varphi=125 \mu \mathrm{m}$, core diameter $\mathrm{d}=5.5 \mu \mathrm{m}$ pitch $\Lambda=5.5 \mu \mathrm{m}$, filling factor $\mathrm{w}=0.9$. In order to achieve these parameters we had to preform an overcladding process, which was conducted on the MCVD lathe. 


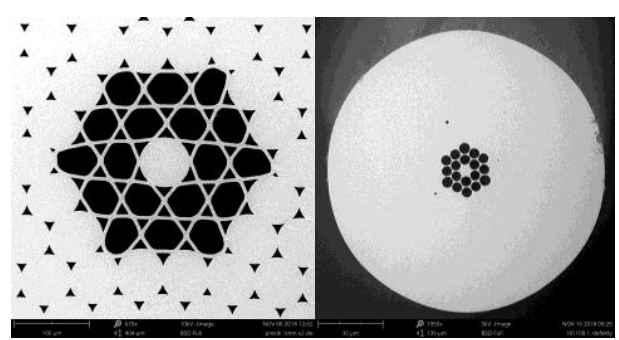

Fig.7. SEM photo of central part of first stage preform (left), crosssection of final fiber (right).

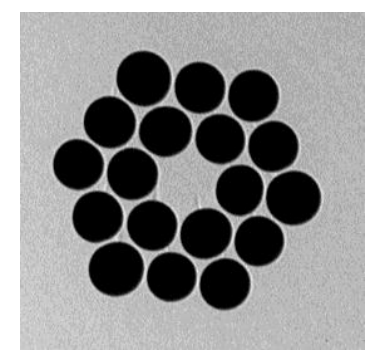

Fig. 8. SEM photo of central part of final fiber.

Our preforms were processed on the drawing tower. In the first process we used the preform made of good quality materials. We experimentally set the following parameters: drawing speed $\mathrm{V}=25 \mathrm{~m} / \mathrm{min}$., force of drawing $\mathrm{F}=0.1 \mathrm{~N}$. In these conditions we drew microstructured fiber with a length of $1100 \mathrm{~m}$. From the second preform made from bad quality materials, fiber was fabricated in very similar conditions. The length of fabricated fiber was also equal to $1100 \mathrm{~m}$. Both fibers possessed very similar geometrical parameters, SEM photos of the high quality preform and fiber are presented in Figs $7 \div 8$.

Fabricated fibers were optically characterised by the Luciol LOR 220 reflectometer at the wavelength $\lambda=1550 \mathrm{~nm}$, the length of measuring impulse was equal $\mathrm{t}=0.25 \mathrm{~ns}$, which gave us more than 10 records at one meter. High resolution allowed us to investigate small and random defects distributed along the fiber. Measured attenuation is presented in Figs $9 \div 10$.

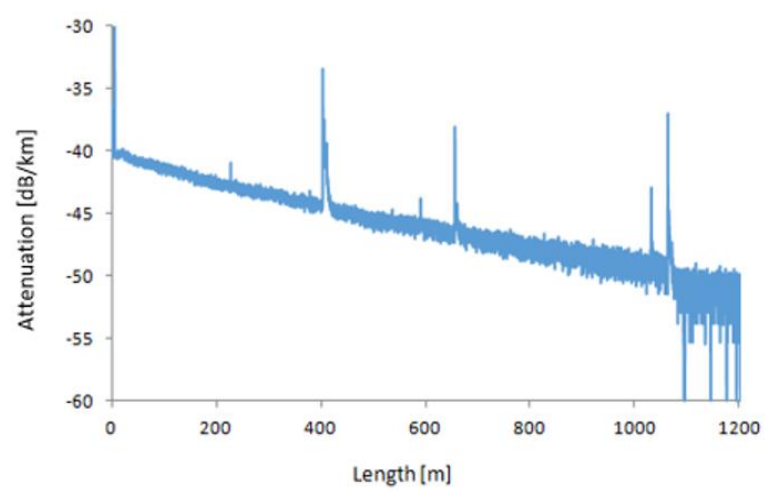

Fig. 9. Attenuation of microstructured fiber made from optimized rods and capillaries.

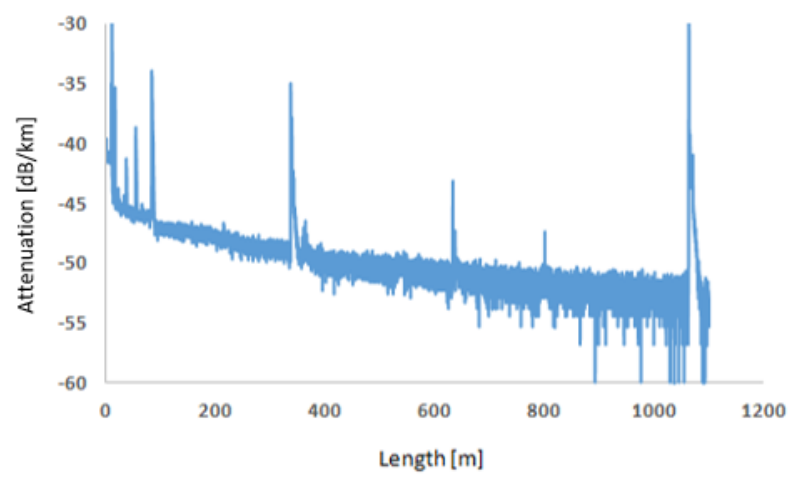

Fig. 1.0 Attenuation characteristic of microstructural optical fiber made of not optimized rods and capillary.

Analyzed attenuation characteristics of two fabricated microstructural fibers showed significant differences. The characteristics of fiber not made from an optimized material showed a high level of noise. At the beginning, after $1=50 \mathrm{~m}$ we registered a large defect and in its close neighbourhood, a series of smaller point defects. Around $1=350 \mathrm{~m}$ we registered another large defect and further several smaller singular point defects. In the case of optimized fiber, the characteristic of attenuation possessed a more regular shape. We did not observe large sections of defects, which were registered at the beginning of nonoptimized fiber. The characteristic was smoother and not so distorted by the noise. Also the number of small point defects was lower. From the characteristic we can point out only one large defect at the $1=400 \mathrm{~m}$.

Non-optimized fiber possessed a large number of bigger and smaller point defects. From its characteristic we also observed a much higher level of noise. The first research showed that the quality of original material capillaries and rods - has a great influence on the fibers quality. In the next step of this research we plan to investigate the mechanisms responsible for these effects.

\section{References}

[1] S. Habib et al., Int. J. Lig. Opt. 124, 3851 (2013).

[2] A. Ziolowicz et al., Proc. SPIE 10130, 101300C (2017).

[3] T.M. Monro et al., Meas. Sci. Technol. 12, 854 (2001).

[4] G. Statkiewicz-Barabach et al., Sensors 17, 1648 (2017).

[5] T. Yoon, M. Bajcsy, Phys. Rev. A 99, 023415 (2019).

[6] A.N. Ghosh et al., J. Opt. Soc. Am. B 35, 2311 (2018).

[7] G. Wójcik et al., Proc. SPIE 10830, 108300H (2018).

[8] F. Xu, Selected topics on optical fiber technology and applications (IntechOpen 2018). 